\title{
Acoustic Data Processing and Transient Signal Analysis for the Hybrid Wing Body 14- by 22-Foot Subsonic Wind Tunnel Test
}

\author{
Christopher J. Bahr, Thomas F. Brooks $\dagger$ William M. Humphreys \\ NASA Langley Research Center, Hampton, Virginia 23681-2199 \\ Daniel J. Stead ${ }^{\top}$ \\ Northrop Grumman
}

\begin{abstract}
An advanced vehicle concept, the HWB N2A-EXTE aircraft design, was tested in NASA Langley's 14- by 22-Foot Subsonic Wind Tunnel to study its acoustic characteristics for various propulsion system installation and airframe configurations. A significant upgrade to existing data processing systems was implemented, with a focus on portability and a reduction in turnaround time. These requirements were met by updating codes originally written for a cluster environment and transferring them to a local workstation while enabling GPU computing. Post-test, additional processing of the time series was required to remove transient hydrodynamic gusts from some of the microphone time series. A novel automated procedure was developed to analyze and reject contaminated blocks of data, under the assumption that the desired acoustic signal of interest was a band-limited stationary random process, and of lower variance than the hydrodynamic contamination. The procedure is shown to successfully identify and remove contaminated blocks of data and retain the desired acoustic signal. Additional corrections to the data, mainly background subtraction, shear layer refraction calculations, atmospheric attenuation and microphone directivity corrections, were all necessary for initial analysis and noise assessments. These were implemented for the post-processing of spectral data, and are shown to behave as expected.
\end{abstract}

\section{Nomenclature}

$\begin{array}{ll}\text { AoA } & =\text { Angle of Attack } \\ B & =\text { normalized signal bandwidth } \\ B E N S & =\text { Broadband Engine Noise Simulators } \\ C S M & =\text { cross-spectral matrix } \\ D & =\text { baffled microphone directivity pattern } \\ D A S & =\text { data acquisition system } \\ E P N L & =\text { effective perceived noise level } \\ F R F & =\text { frequency response function } \\ G & =\text { one-sided power spectral density } \\ H W B & =\text { Hybrid Wing Body } \\ J_{()} & =\text {Bessel function of the first kind, order }() \\ K & =\text { Kullback-Leibler divergence } \\ k & =\text { acoustic wavenumber } \\ M & =\text { Mach number }\end{array}$

\footnotetext{
*Research Aerospace Engineer, Aeroacoustics Branch, MS 461, Senior Member AIAA, christopher.j.bahr@nasa.gov †Senior Research Scientist, Aeroacoustics Branch, MS 461, Fellow AIAA

${ }^{\ddagger}$ Senior Research Engineer, Advanced Sensing \& Optical Measurement Branch, MS 493, Senior Member AIAA

$\S$ Research Student Trainee, Aeroacoustics Branch, MS 461, Student Member AIAA

ฯSenior Engineer, MS 461
} 


\begin{tabular}{|c|c|}
\hline$N$ & $=$ number of samples in a block of data \\
\hline$P$ & $=$ probability distribution function \\
\hline$p$ & $=$ probability density function \\
\hline$Q$ & $=$ probability distribution function, estimate of $P$ \\
\hline$q$ & $=$ probability density function, estimate of $p$ \\
\hline$R$ & $=$ microphone diaphragm radius \\
\hline rms & $=$ root-mean-square \\
\hline$y_{n}$ & $=$ individual sample in a block of data \\
\hline$\alpha$ & $=$ gamma distribution shape parameter \\
\hline$\beta$ & $=$ gamma distribution scale parameter \\
\hline$\Gamma$ & $=$ gamma function \\
\hline$\gamma$ & $=$ incomplete gamma function \\
\hline$\theta$ & $=$ wave angle of incidence, referenced to microphone diaphragm normal \\
\hline$\nu$ & $=$ effective degrees of freedom of a signal of block size $N$ \\
\hline$\sigma^{2}$ & $=$ variance of a block of data \\
\hline$\chi_{N}^{2}$ & $=$ sum of the squares of the samples in a block of data \\
\hline
\end{tabular}

\section{Introduction}

NASA's Hybrid Wing Body (HWB) N2A-EXTE acoustic test in Langley Research Center's 14- by 22Foot Subsonic Wind Tunnel was a large-scale experiment to demonstrate potential technologies to reach the Environmentally Responsible Aviation Project's N+2 system level aircraft noise goal of 42 cumulative

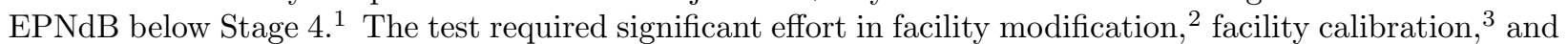
instrumentation. ${ }^{4}$ The outcome of the test supports analysis of turbomachinery ${ }^{\sqrt{5}}$ and jet noise $e^{6}$ shielding, airframe noise and system noise..$^{7}$

Analysis requirements drove the test to involve approximately 2,000 unique arrangements of the model, instrumentation and operational parameters (an example is shown in Fig. 1), leading to the acquisition of a large amount of aeroacoustic data. $\frac{8}{}$ Initial results were desired in near-time, allowing for potential decisions regarding additional test configurations. Post-test processing required the development and use of multiple types of acoustic corrections to the data to reconstruct best estimates of lossless, free-field measurements. These corrections range in complexity from background noise subtraction to analysis and rejection of transient hydrodynamic contamination of individual microphone time series.

This paper presents the data flow of the acoustic processing for the initial analysis of the test results. The transient rejection technique is shown to qualitatively reject obvious contamination, while retaining data of interest. Individual microphone, spectral-level corrections which are common to most of the initial data processing are discussed, with some characteristic results provided.

\section{Initial Data Acquisition \& Processing}

Details of the data acquisition hardware are provided elsewhere $\sqrt[214]{4}$ In brief, voltage signals from the microphones and surface pressure sensors were transmitted through various hardware-appropriate amplifiers, filters and power supplies, some controlled automatically by the data acquisition system (DAS) and some set manually. These voltage signals were acquired by the DAS, a 192-channel 3-unit assembly of National Instruments (NI) PXI-1045 chassis populated with 16-bit NI PXI-6120 digitizers. For any given set of model-traverse configurations, the individual channel gains were set such that the observable dynamic range was maximized within the limits defined by the 16-bit system, while avoiding over-ranging. These settings were matched between data acquisition from noise sources of interest and corresponding background noise acquisitions, so any background noise subtraction would maintain proper scaling for both pressure and voltage signals (see Section IV.A). To maximize system throughput, time series data were streamed to a RAID system as raw 16-bit integer data from the DAS, with appropriate metadata for conversion from integers to volts stored in the header of each time series binary file.

In-situ data processing had two somewhat competing requirements: accessibility and efficiency. Time series stored in a custom binary format allow for efficient disk and memory utilization, but require custom code with knowledge of the format, often impeding accessibility. Data stored in, for example, ASCII format,

2 of 16

American Institute of Aeronautics and Astronautics 


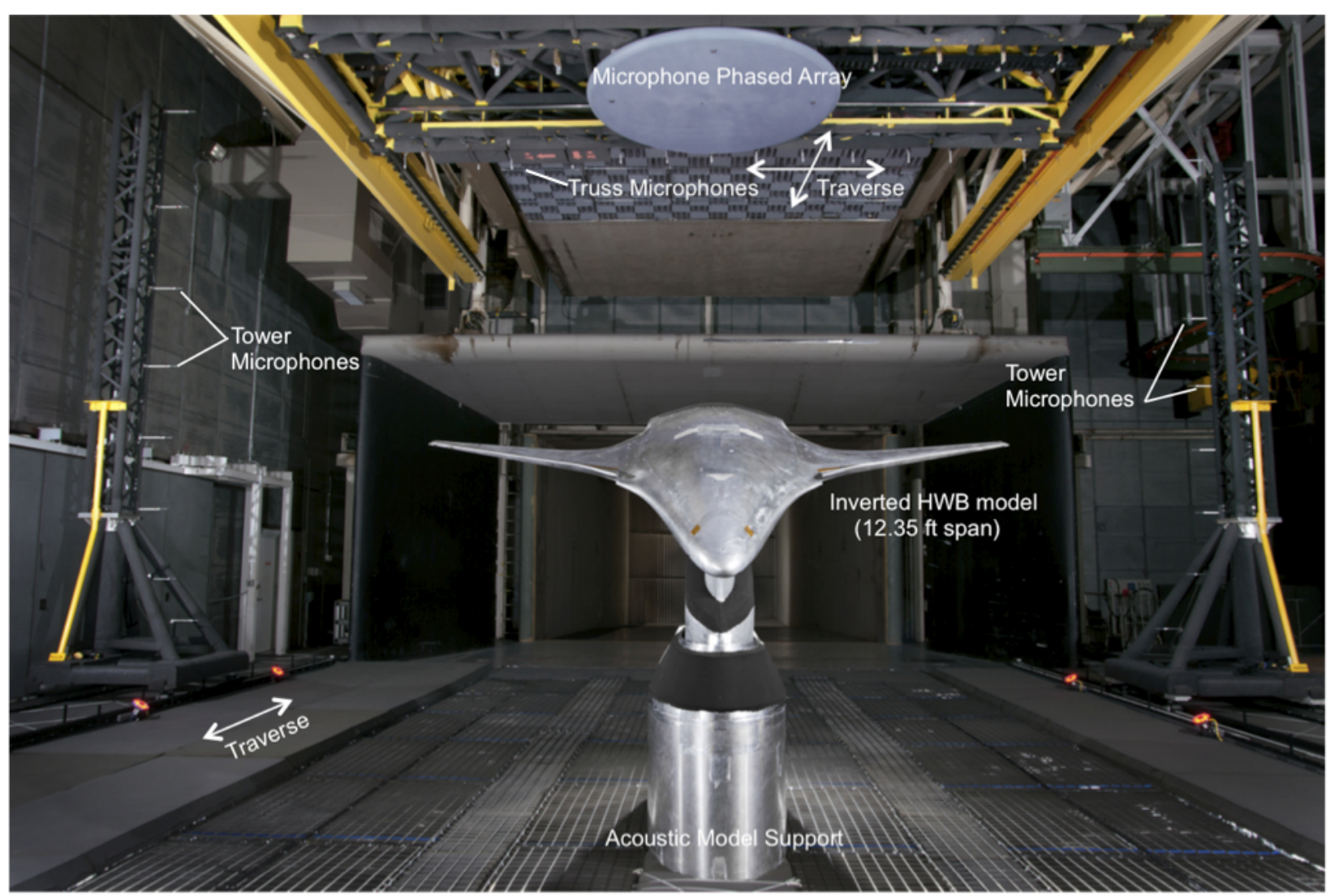

Figure 1. Example arrangement of the HWB model, phased array and tower traverses.

is generally highly accessible but very expensive in terms of file size. Prior to the test, it was decided that a common data format tailored for large-scale scientific data was an appropriate archival tool. Specifically, the Network Common Data Form (NetCDF) was selected ${ }^{9}$ A post-acquisition code was written to convert the custom binary data to the more accessible NetCDF format. This code was written in a combination of National Instruments LabVIEW ${ }^{10}$ and C. It stored appropriate DAS information, along with facility and model data, in the header and as variables of NetCDF files for every time series, along with the 16-bit integer time series data itself.

In parallel, a code to compute the cross-spectral matrix (CSM) of each acquisition was required. Historically this task has been offloaded to a Fortran code operating on a cluster, and has not had a sufficiently-short turnaround time to allow for near-time data analysis. The cluster-based Fortran code was replaced with a MATLAB implementation operating on a local workstation. The effort of computing the Fourier transforms and vector outer products of each block of data to construct an average CSM was offloaded to an NVIDIA Tesla C1060 GPU installed in the workstation, as GPU computing has native language constructs in MATLAB! ${ }^{11}$ CSMs were stored in NetCDF files with facility, model, and processing information.

\section{Work Flow Architecture}

The processing workflow in the construction of CSMs from the acquired time series data was as follows (illustrated in Fig. 2):

1. Load the 16-bit integer time series data.

2. Convert the data to single-precision floating-point format.

3. Use the DAS range information to scale the data from integer counts to volts, as observed by the DAS.

4. Use the amplifier gain settings to scale the data from volts as observed by the DAS to volts output from the transducer. 
5. Use the transducer sensitivity database to scale the time series data from volts to pascals.

6. Check the transducer type. If it is a microphone flush-mounted in the array plate, divide the pressure signal by two to account for pressure doubling on the plate face.

7. Split the data into blocks of 8192 points. Subtract the block mean, apply a Hamming window, and Fourier transform the data.

8. Apply the appropriate broadband level correction for the window function and correct for the transducer frequency response function (FRF).

9. Compute one-sided auto- and cross-spectra through the traditional RMS averaging procedure. 12

Normally, data were acquired at a sampling rate of $250 \mathrm{kHz}$ for just over 30 seconds, yielding 920 blocks of data. For a block length of 8192 points, the resultant CSMs had bin widths of $30.5 \mathrm{~Hz}$.

\section{Implementation}

In practice, many of these steps were combined to maximize throughput. The executed workflow was:

1. Pre-compute all of the scalar corrections required to convert integer time series data to pressure time series data, for every transducer.

2. Multiply these scalar corrections by a Hamming window for every transducer, and transfer this array of scaled window functions to the GPU.

3. Initialize an all-zeros CSM of complex single-precision on the GPU.

4. For every block of data:

- Transfer the 16-bit integer time series blocks for every transducer for the given block number to the GPU.

- Re-cast the data to single-precision floating-point format.

- Element-by-element multiply the array of blocks by the array of window functions.

- Batch-FFT the array of data blocks.

- Compute the outer product of the vector of transducer Fourier coefficients for each frequency and add to the initialized CSM.

5. Transfer the CSM back to workstation memory.

6. Scale the CSM to account for the window function broadband correction, number of blocks, and transducer FRFs.

The modified process reduced CSM computation time to well under a minute, with most of the time spent transferring the data across the control room network. The majority of this improvement was due to efficient use of the strengths of GPU computing, which alone accounted for an order-of-magnitude reduction in the processing time when compared to an equivalent CPU-based process executed on the workstation.

\section{Post-Test CSM Reconstruction: Transient Analysis}

The in-situ CSMs were treated as approximates. A refined analysis was discussed and implemented posttest. Simple changes included an upgrade of the processing workstation with an NVIDIA Kepler K20c GPU, implementation of a correction for the Precision Filter FRFs (incorporated at the same stage of processing as the transducer FRFs), and an option to compute autospectra only, in order to minimize memory and disk storage when cross-spectra are unnecessary.

A more complex change involved the assessment of the data for transient contamination. NASA Langley's 14- by 22-Foot Subsonic Wind Tunnel is, by design, an aerodynamic wind tunnel which can operate in an open test section configuration. While significant acoustic improvements have been applied to the facility,

$$
4 \text { of } 16
$$




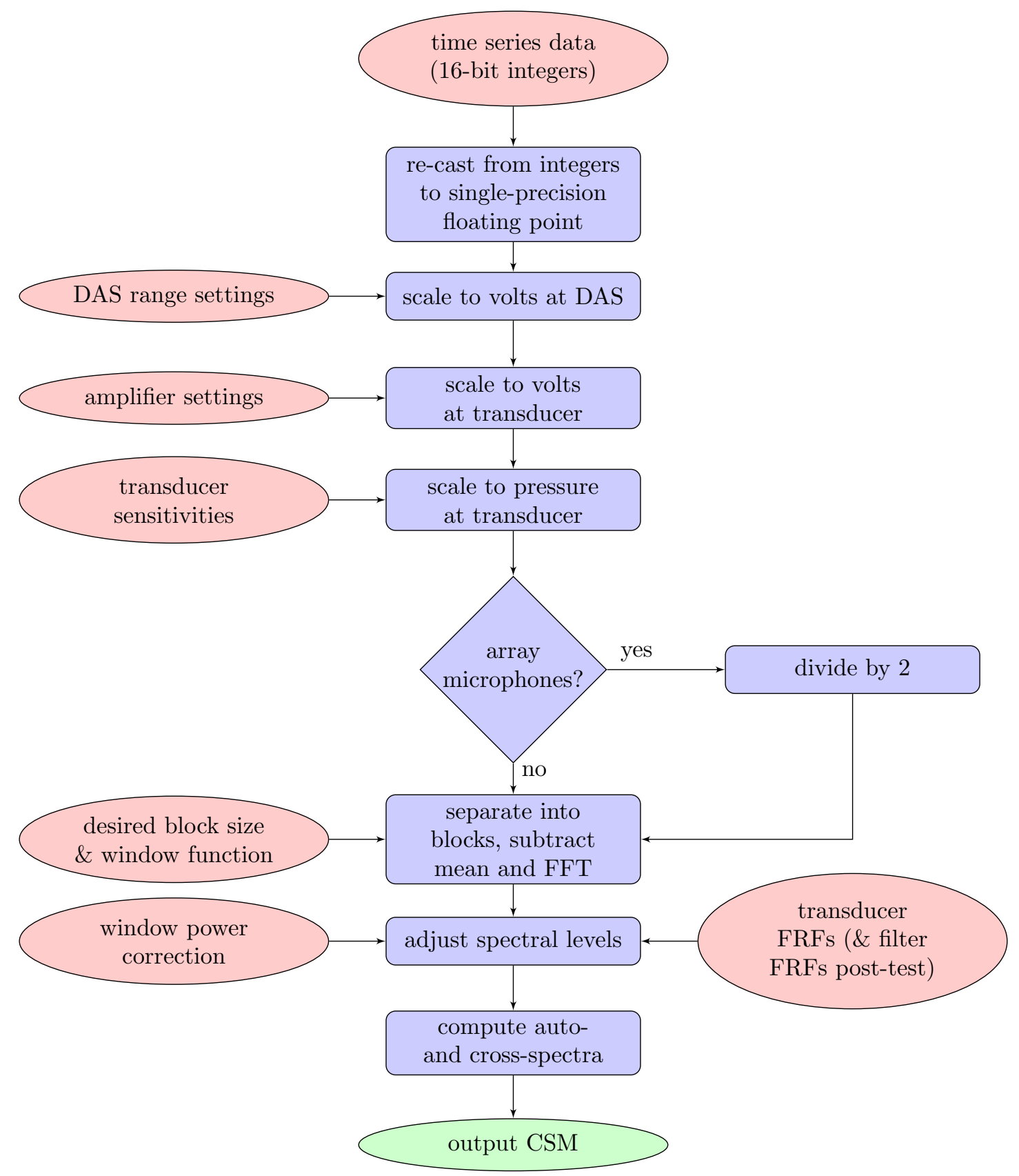

Figure 2. Work flow architecture to compute the CSM for a given acquisition. 
measurement microphones are, under some installation configurations, close enough to the open-jet shear layer that hydrodynamic gusts may contaminate the out-of-flow acoustic measurements. This was primarily observed when microphones were at the far-downstream end of the test section, although occasional gust impingement was seen at other measurement stations.

An extreme example of this from the airframe noise component of the test is shown in Fig. 3 . The plotted data are for an acquisition where one of the speakers embedded in the model body ${ }^{2}$ is driven with a random bandpass signal spanning $4 \mathrm{kHz}$ to $16 \mathrm{kHz}$. The HWB model is pitched to an angle of attack (AoA) of $14.5^{\circ}$, and the test section Mach number is $M=0.23$. The acoustic hardware is traversed to the far-downstream end of the test section. As shown by the time series in Fig. 3(a), the array center microphone signal appears as might be expected for a stationary, band-limited random signal. The south tower microphone, located in the upper-right-hand corner of the picture in Fig. 1. clearly experiences extreme random bursts as shown in Fig. 3(b). The corresponding autospectra are shown in Figs. 3(c) and 3(d). While the array center microphone spectrum shows the low frequency content of the signal at $4 \mathrm{kHz}$, the south tower microphone spectrum is masked by the low frequency content of the burst. Note that at this stage of processing, two clean signals would not overlay due to differences in propagation distance between the source and each microphone, speaker directivity, and additional effects to be discussed later.

The south tower microphone time series is partitioned into 920 blocks, each of length 8192 points, and a histogram of the block variances is computed. This histogram is shown with respect to the left axis in Fig. 4. with the 16 most energetic blocks removed from the plot. Even without these blocks, which would extend the $\mathrm{x}$-axis beyond a variance of $500 \mathrm{~Pa}^{2}$, this histogram shows a long, thin tail in the direction of large variance values. While the contamination from high-variance blocks is obvious through visual inspection of the time series and can be dealt with by manually evaluating the data and rejecting blocks, applying such a procedure to roughly 250,000 time series records is intractable. A novel, automated process for analyzing the data is required.

Several methods were considered for separating the data. Initially, visual inspection suggested that a voltage threshold could be used in separating the data. However this was somewhat arbitrary and specific to each run condition. A method evaluating histogram shape was then evaluated. Here, data histograms of block rms levels were constructed using sub-blocks 1/8th the size of the full block length (1024 points), allowing for more converged behavior of the histograms. Histograms from known clean microphone signals for a given test condition, for example the array center microphone positioned upstream in the test section, were also computed. The ratio between the peak sub-block rms and the rms at the histogram peak, or the mode rms, was computed to give a sense of the span of the high-variance tail of the histogram. The ratio for the reference histogram was compared to the ratio for the contaminated histogram, and sub-blocks with rms ratios beyond this reference ratio were flagged. Any full block containing a flagged sub-block was rejected. This technique was successful in identifying and eliminating block contamination, although the requirement of a reference signal required the assumption that for every run condition an appropriate reference was known. A process which avoided the use of a reference signal was considered and selected for the final analysis, which follows.

The core of the selected rejection process is the assumption that the underlying acoustic signal of interest is a stationary, zero-mean, gaussian random process. If the acoustic samples, $y$, are truly gaussian-distributed random variables with zero mean and unit variance, then the sum of the squares

$$
\chi_{N}^{2}=\sum_{n=1}^{N} y_{n}^{2},
$$

of a set of $N$ samples is a random variable itself, following a chi-square distribution with $N$ degrees of freedom $\frac{13}{13}$ If both sides of this relation are divided by $N$, this shows that for $N$ samples, or for data blocks of length $N$, the block variance can be related to the sum of the squares,

$$
\sigma^{2}=\frac{\chi_{N}^{2}}{N}=\frac{1}{N} \sum_{n=1}^{N} y_{n}^{2} .
$$

This also follows a chi-square distribution, by simply normalizing the data values $y_{n}^{2}$ by $N$. Note that for finite $N$ this relation involved a biased estimator of the block variance. An unbiased estimator can be used by substituting $N-1$ for $N$ in the normalization. 


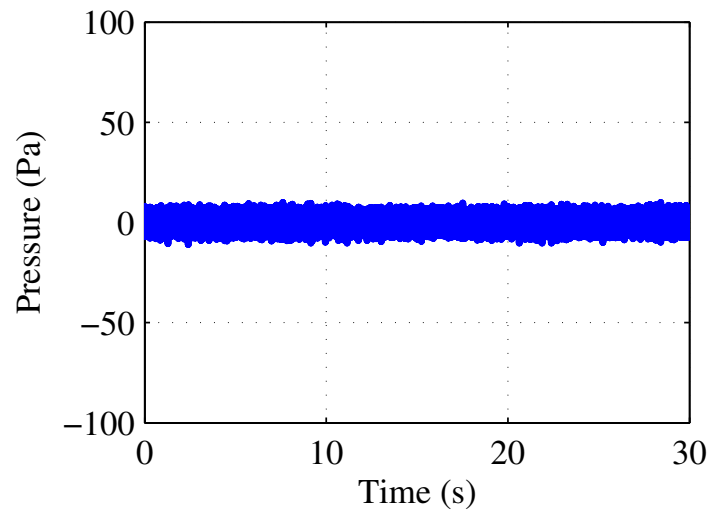

(a) Array center microphone time series.

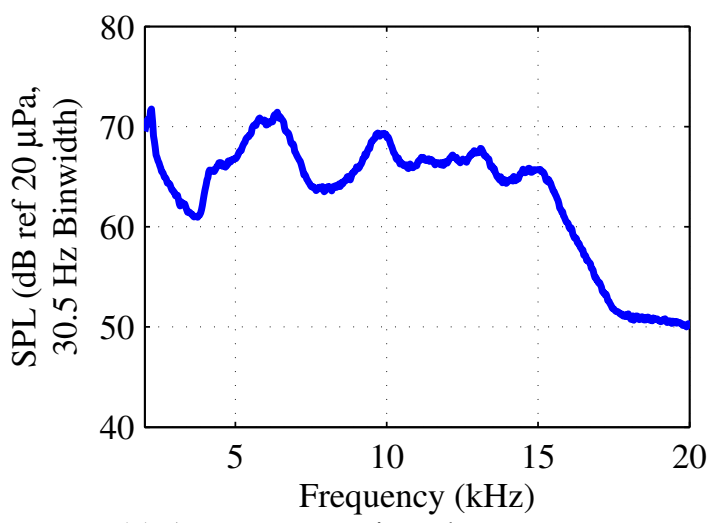

(c) Array center microphone autospectrum.

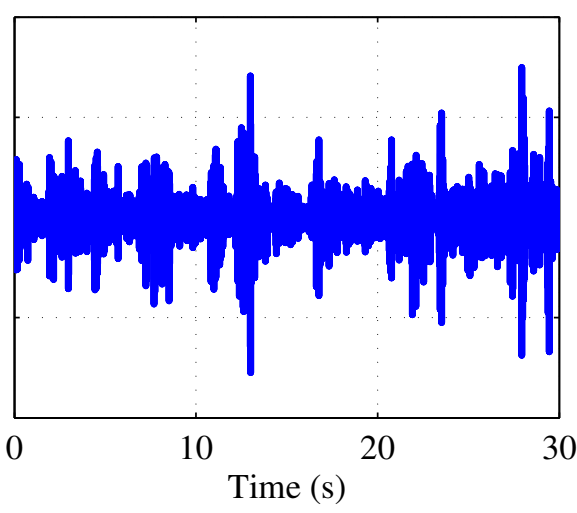

(b) South tower microphone 7 time series.

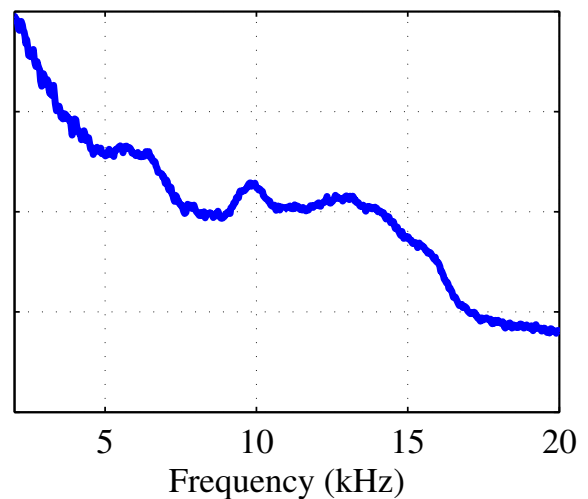

(d) South tower microphone 7 autospectrum.

Figure 3. Example data contamination by hydrodynamic impingement. The two compared microphones observe a calibration signal with an output band of $4 \mathrm{kHz}$ to $16 \mathrm{kHz}$, emitted by one of the model embedded speakers. The HWB model is at $14.5^{\circ} \mathrm{AoA}$, and the test section Mach number is $M=0.23$. Acoustic hardware are at the far downstream traverse location.

In practice, the acoustic signal is not truly random, but has a finite bandwidth. This normalized bandwidth, $B$, alters the effective degrees of freedom, $\nu$, of the signal ${ }^{14}$ For example, a block of 8192 samples of a signal which is truly random has a spectrum of white noise and a bandwidth of $100 \%$, so $\nu=N=8192$. If the signal passes through an ideal lowpass filter set to $50 \%$ of the sampling bandwidth, or $B=0.5$, the effective degrees of freedom is $\nu=B \times N=4096$. The fractional, normalized bandwidth can be estimated through a simple procedure. First, the one-sided power spectral density of the signal must be computed. This function of frequency, $G_{y y}(f)$, must then be normalized such that its peak is unity,

$$
G_{y y, \text { norm }}(f)=\frac{G_{y y}(f)}{\max \left[G_{y y}(f)\right]} .
$$

The average of this normalized spectral density is then computed by integrating across the measurement bandwidth and dividing by the span,

$$
B=\frac{1}{f_{\max }} \int_{0}^{f_{\max }} G_{y y, \text { norm }}(f) \mathrm{d} f
$$

While the acoustic data sample distribution does have a mean of approximately zero due to the use of a high pass filter during data acquisition, the variance of the distribution for $y$ is unknown. A more general distribution is necessary to model the block variance, $\sigma^{2}$. As a generalization of the chi-square distribution, 


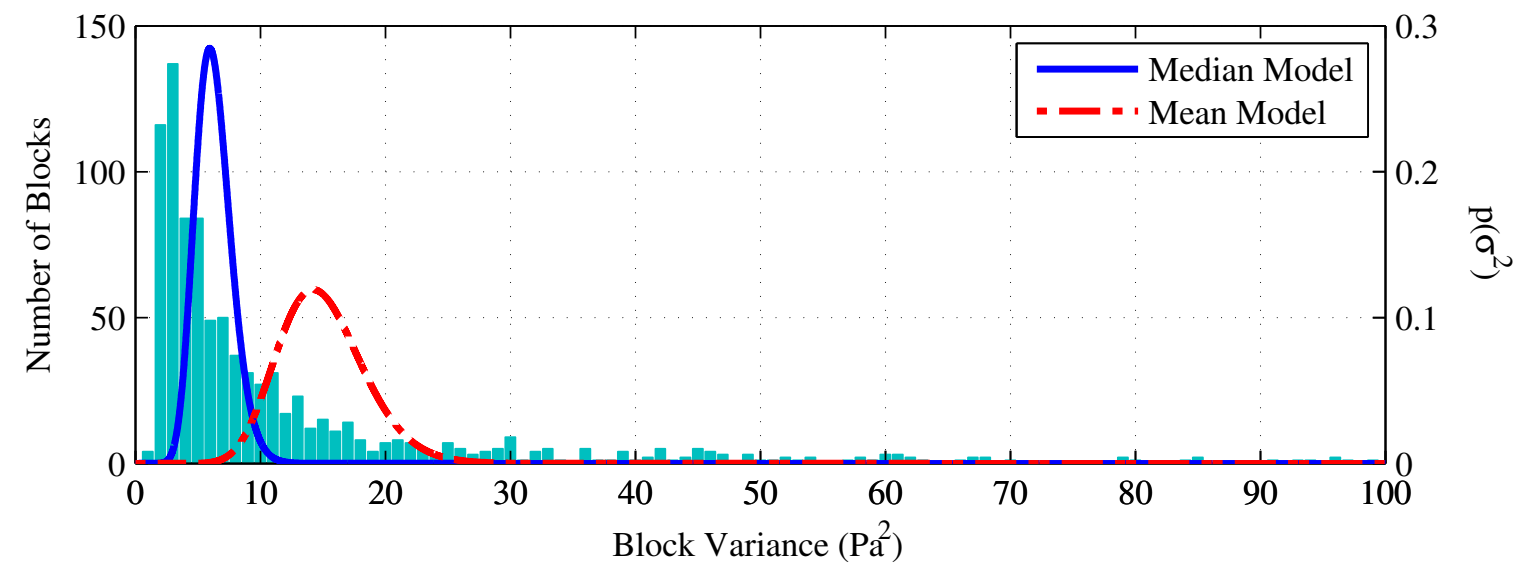

Figure 4. Histogram of block variances from the south tower time series in Fig. 3 excluding the 16 most energetic blocks, and modeled data probability density functions.

the gamma distribution can be used.15 The probability density function for a gamma distribution of the block variance (with a zero location parameter) is given by

$$
p\left(\sigma^{2}\right)=\frac{1}{\beta \Gamma(\alpha)}\left(\frac{\sigma^{2}}{\beta}\right)^{\alpha-1} e^{\frac{-\sigma^{2}}{\beta}},
$$

where $\alpha=\nu / 2$ is the shape parameter, $\beta$ is the scale parameter and $\Gamma$ is the gamma function

$$
\Gamma(\alpha)=\int_{0}^{\infty} t^{\alpha-1} e^{-t} \mathrm{~d} t .
$$

For $\beta=2$ this collapses to the chi-square distribution. This scale parameter allows a distribution fit to handle non-unity variance of $y$.

As previously stated, the effective degrees of freedom and thus the shape parameter of a distribution fit can be derived from the signal bandwidth, which can be approximated from a spectral estimate of the signal. The scale parameter must now be determined. An easy, if biased, $\frac{16}{16}$ estimate of $\beta$ can be obtained from its maximum likelihood estimator

$$
\beta=\frac{\overline{\sigma^{2}}}{\alpha}
$$

where $\overline{\sigma^{2}}$ is an estimate of the mean of the block variances. When evaluating the mean of the block variances, however, it is readily observable that this mean will be sensitive to the high-variance, hydrodynamicallycontaminated blocks. A statistic less sensitive to extreme values is necessary for computing $\beta$. One such statistic is the median of the block variances. The median occurs where the probability distribution function is 0.5 . The probability distribution function for the gamma distribution is given by

$$
P\left(\sigma^{2}\right)=\frac{\gamma\left(\alpha, \frac{\sigma^{2}}{\beta}\right)}{\Gamma(\alpha)},
$$

where $\gamma$ is the (non-normalized) incomplete gamma function 15

$$
\gamma\left(\alpha, \frac{\sigma^{2}}{\beta}\right)=\int_{0}^{\frac{\sigma^{2}}{\beta}} t^{\alpha-1} e^{-t} \mathrm{~d} t .
$$

The equation for the median variance is thus

$$
\frac{1}{2}=\frac{\gamma\left(\alpha, \frac{\sigma_{\mathrm{med}}^{2}}{\beta}\right)}{\Gamma(\alpha)} .
$$

$$
8 \text { of } 16
$$


Software libraries exist for efficiently inverting $\gamma$ for a given $\alpha$, thus yielding an estimate of the median variance normalized by $\beta$. The experimental median variance can then be divided by this estimate, yielding an estimate of $\beta$. Thus for a given shape factor $\alpha$, two scale factors can be readily computed from the data. One, $\beta_{\text {mean }}$, is based on the mean of the block variances and susceptible to extreme values of contamination. The other, $\beta_{\text {median }}$, is based on the median of the block variances, and is less susceptible to extreme values.

Having two scale factors allows for the construction of two gamma distributions. These can be compared to gain some sense of the relative influence of extreme block variances on the data set. Visually this is done in Fig. 4 with respect to the right axis. One numeric metric for comparing distributions is the Kullback-Leibler divergence $\frac{17}{17}$ The divergence $K$ is a measure of the information lost when probability distribution $Q$ (or density $q$ ) is used to estimate distribution $P$ (or density $p$ ). This is expressed as

$$
K(p \| q)=\int \ln \left[\frac{p\left(\sigma^{2}\right)}{q\left(\sigma^{2}\right)}\right] p\left(\sigma^{2}\right) \mathrm{d}\left(\sigma^{2}\right) .
$$

While in general this can be difficult to compute, it is greatly simplified in the case of two gamma distributions with a common $\alpha$. In this case, some manipulation yields

$$
K(p \| q)_{\alpha_{p}=\alpha_{q}=\alpha}=\alpha\left(\ln \beta_{q}-\ln \beta_{p}+\frac{\beta_{p}-\beta_{q}}{\beta_{q}}\right),
$$

or as used in this application

$$
K\left(p_{\text {median }} \| p_{\text {mean }}\right)_{\alpha_{\text {median }}=\alpha_{\text {mean }}=\alpha}=\alpha\left(\ln \beta_{\text {mean }}-\ln \beta_{\text {median }}+\frac{\beta_{\text {median }}-\beta_{\text {mean }}}{\beta_{\text {mean }}}\right) .
$$

To summarize, two data distributions can be estimated. The distribution based on the block variance mean is more sensitive to high-variance contamination than the distribution based on block variance median. A metric is constructed for comparing the two distributions. Now a procedure is proposed for determining which blocks of a given time series to retain, and which to reject. It should be noted here that for the number of blocks used, converged data distributions are not expected. The intent of the following procedure is to provide an automated engineering tool to locate contaminated blocks in the time series, not to accurately estimate the probability distribution of the acoustic data block variance.

First, every microphone time series is broken into 920 blocks of desired length $N=8192$ points. The variance of each block is computed, and the blocks are sorted by their variance, from low to high. The initial, 100 lowest-variance blocks are selected. An autospectral density is computed from this set of low-variance blocks, and used to compute $\alpha$. $\beta_{\text {mean }}$ and $\beta_{\text {median }}$ are computed, followed by $K$. The next block, in order of ascending variance, is added to the set of blocks and the process repeated. This continues until all of the blocks of data have been used, producing $K$ as a function of block count in order of ascending variance. The block count of minimum $|K|$ is selected as the cut-off. Sorted blocks above this are rejected.

As stated, 100 blocks comprise the initial lowest-variance block set. This minimum provides a safeguard should the automated algorithm attempt to reject too many blocks. When computing isolated autospectra, each time series is handled independently with the 100 block minimum. For CSM construction, if a block is rejected for one channel of the system it is rejected for all channels of the system. This may allow a CSM to drop below 300 blocks, which based on prior experience has been suggested as a minimum requirement for converged cross-spectral data. If this is the case, the CSM is considered sufficiently contaminated that the rejection procedure may not improve the results. For such a situation (highly infrequent), the rejection technique is disabled, the condition logged in the CSM file header, and all data blocks are used.

The transient rejection procedure is applied to the south tower microphone data from Fig. 3(b). Of the 920 input blocks, 567 are rejected. The histogram of the remaining block variances is shown in Fig. 5. along with the median- and mean-based probability density functions for the retained block set. As expected, the probability density functions overlay for the minimum value of $|K|$. The output of the procedure is shown in Figs. 6(a) and 6(b). Visually, the technique has identified and removed the obvious contamination from the time series. In the spectral analysis, the $4 \mathrm{kHz}$ content of the signal is now visible, with a reduction of up to $10 \mathrm{~dB}$ in the microphone autospectrum at lower frequencies. Higher frequencies are unaffected. These results indicate that transient signal analysis and rejection may be critical in the use of potentially-contaminated data sets. 


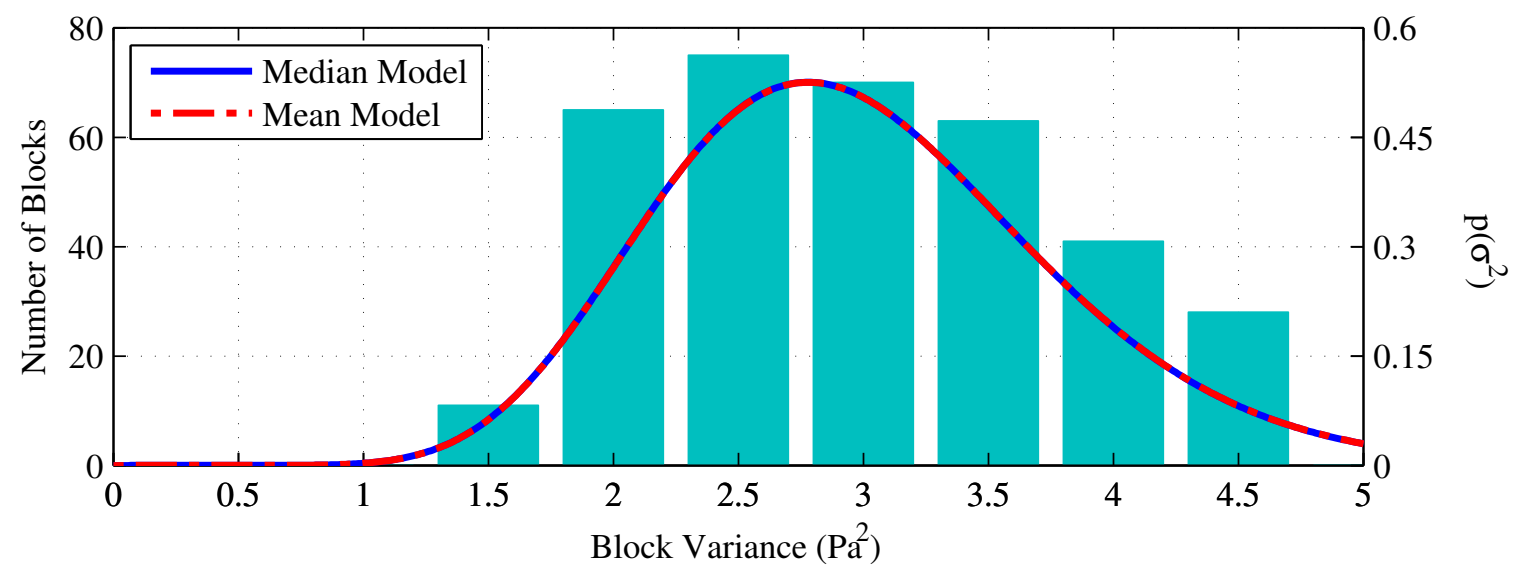

Figure 5. Post-rejection histogram of south tower time series data from Fig. 4, along with postrejection models (mean model near-completely overlays the median model).

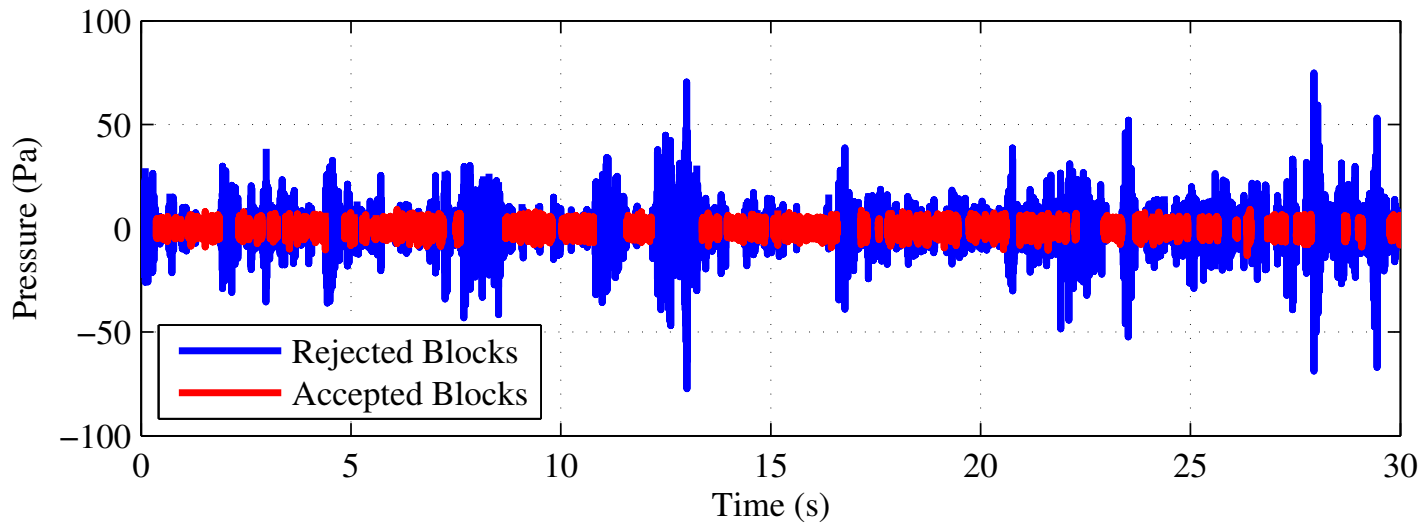

(a) Blocks accepted and rejected by the algorithm.

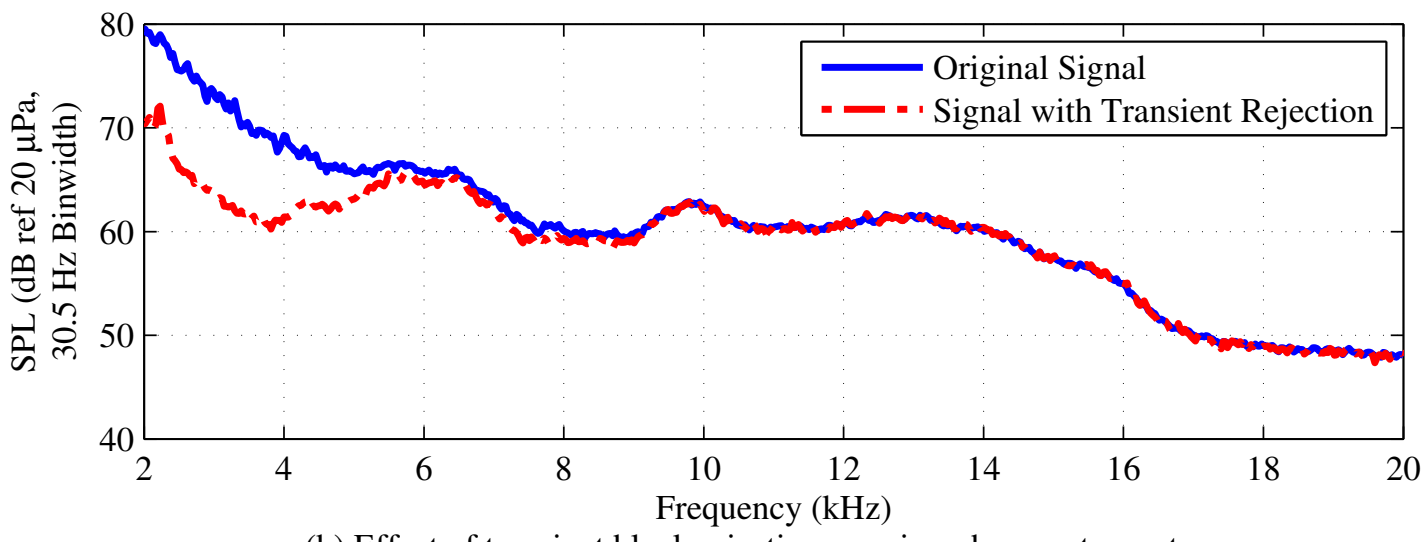

(b) Effect of transient block rejection on microphone autospectrum.

Figure 6. Results of transient rejection algorithm when applied to the south tower time series data from Fig. 3(b). Data blocks are plotted as a function of time. The shift in the estimated data autospectrum is shown. 


\section{Post-Test Data Corrections}

A series of data corrections are often necessary prior to application and interpretation of the autospectral data from the HWB wind tunnel test. All of these corrections are applied on a narrowband basis. In autospectral analysis, all of the corrections aside from background subtraction assume a well-defined acoustic source location. At frequencies where this is not the case, either due to ill-defined acoustic source regions or the dominance of extraneous noise, these techniques would be unreliable.

For beamforming and deconvolution analysis the procedure is similar. However, every correction which must assume a source location instead generates a set of corrections for every combination of beam map grid points and microphones, and for every frequency bin.

\section{IV.A. Background Subtraction}

Background subtraction is intended to remove extraneous noise from that of the signal of interest. It is performed without regard to an acoustic source location. Background subtraction is a simple data correction, which has been used repeatedly for previous NASA Langley aeroacoustic experiments $\frac{18}{20}$ Here, auto- and cross-spectra of a facility measurement without the acoustic source of interest are subtracted vectorially from auto- and cross-spectra of a facility measurement with the acoustic source of interest. As stated previously, this is done for runs with matched acquisition settings (identical ranges and gains), so both voltage and pressure noise are scaled properly between the two measurements. Subtraction occurs in the power domain with units $\mathrm{Pa}^{2}$ for both the real and, for the CSM off-diagonal cross-spectra, imaginary components of the spectra.

In application, normally background subtraction assumes that there is no correlation between the background noise measurement and the source of interest, so any strong interaction effects may interfere with this procedure. This correction is the first of the post-processing corrections applied, as it makes no assumption about the nature of the acoustic field beyond the independence of the background noise from the source of interest. Often in low signal-to-noise ratio frequency ranges, the background noise level for certain frequency bins for the autospectra may be equal to or greater than the noise from the source of interest. In this case, the power for the bin is often set to zero in applications, the bin flagged for user notification, and the bin not considered in subsequent analysis. At user discretion for spectra where this happens to a significant number of bins in frequency bands of interest, background subtraction may be excluded from the data processing. For the cross-spectral terms, there is no distinct error determination measure, such as positive or negative sign. Applications determine that. For example, CSM-based array beamforming may be substantially immune from low signal-to-noise issues.

A simple example of background subtraction for autospectra is illustrated in Fig. 7. Here, the acoustic hardware were traversed to the far-downstream location and the tunnel operated at $M=0.17$. Model airframe noise, along with the tunnel wind noise, were included in the data acquired. A model-embedded speaker was then turned on, and data were acquired again. Background subtraction extends the range of observable speaker input signal at lower frequencies. At higher frequencies, it extends the observable range of the speaker output. Note that the speaker input range was 4 to $16 \mathrm{kHz}$, but system non-linearities extended the noise output range. Power bins which are set to zero, near $3 \mathrm{kHz}$, are not plotted. Near-zero powers such as the trough near $37 \mathrm{kHz}$ are seen.

\section{IV.B. Shear Layer Correction}

In open-jet test section aeroacoustic wind tunnel testing, shear layer correction is necessary for analysis of the data. While the overall level correction to the data may be minor at low Mach numbers, the shear layer plays a significant role in the path traveled by a packet of acoustic energy. For this study, Amiet's shear layer correction method is used ${ }^{21}$ Here, the facility shear layer sides are modeled as planes, and an acoustic source location must be defined. This source location is determined by the particular test configuration. The shear layer correction technique determines the transmission path for a packet of acoustic energy traveling from the source to an observer. Additionally it calculates the emission coordinates for the source-observer position, which follow the phase fronts of the acoustic waves emitted from the source and correspond to the emission angles from an in-flight source passing an observer. An example of a shear layer analysis for a test case dominated by the main landing gear airframe noise is shown in Fig. 8. This figure shows the correction for a microphone located above the model. From Fig. 1, it is evident that there are three planar 


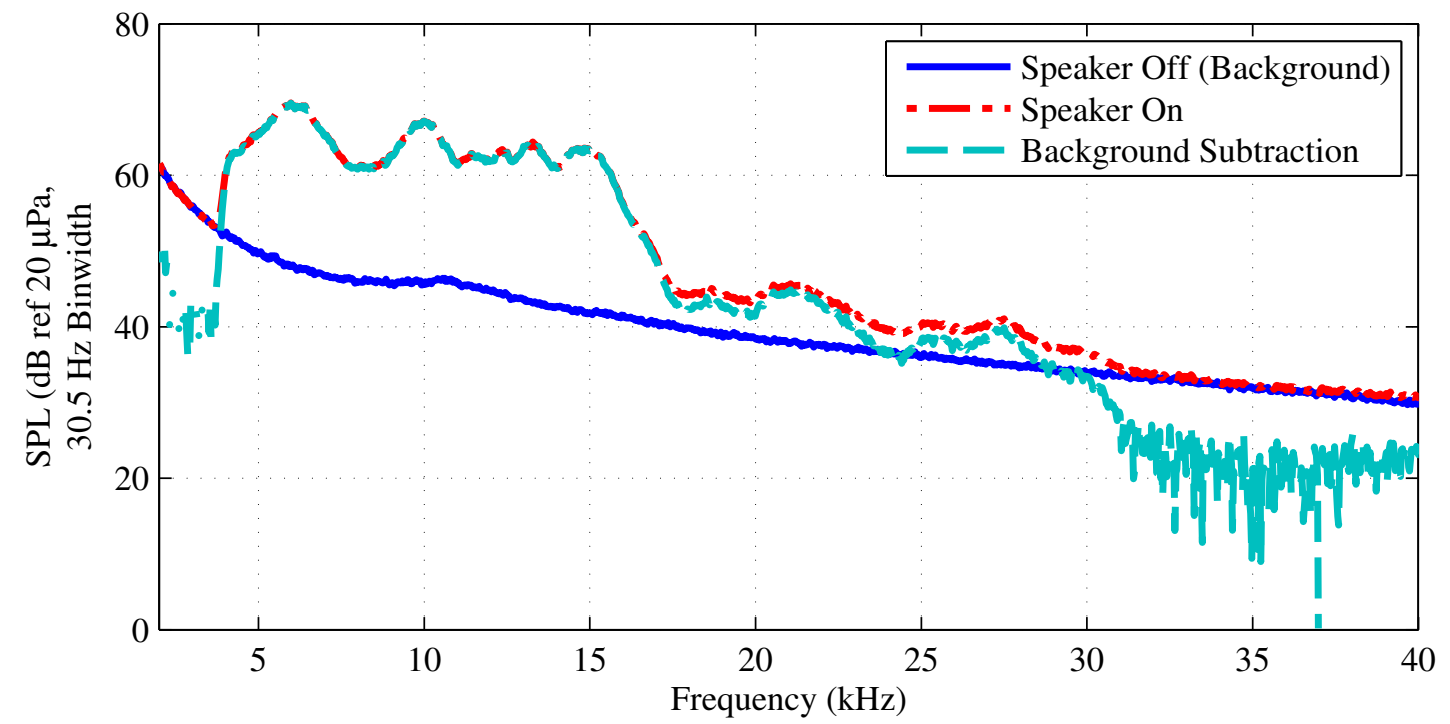

Figure 7. Example of background subtraction process applied to the array center microphone observing a model embedded speaker. The speaker signal has an output band of $4 \mathrm{kHz}$ to $16 \mathrm{kHz}$. The HWB model is at $14.5^{\circ}$ AoA, and the test section Mach number is $M=0.17$. Acoustic hardware are at the far downstream traverse location.

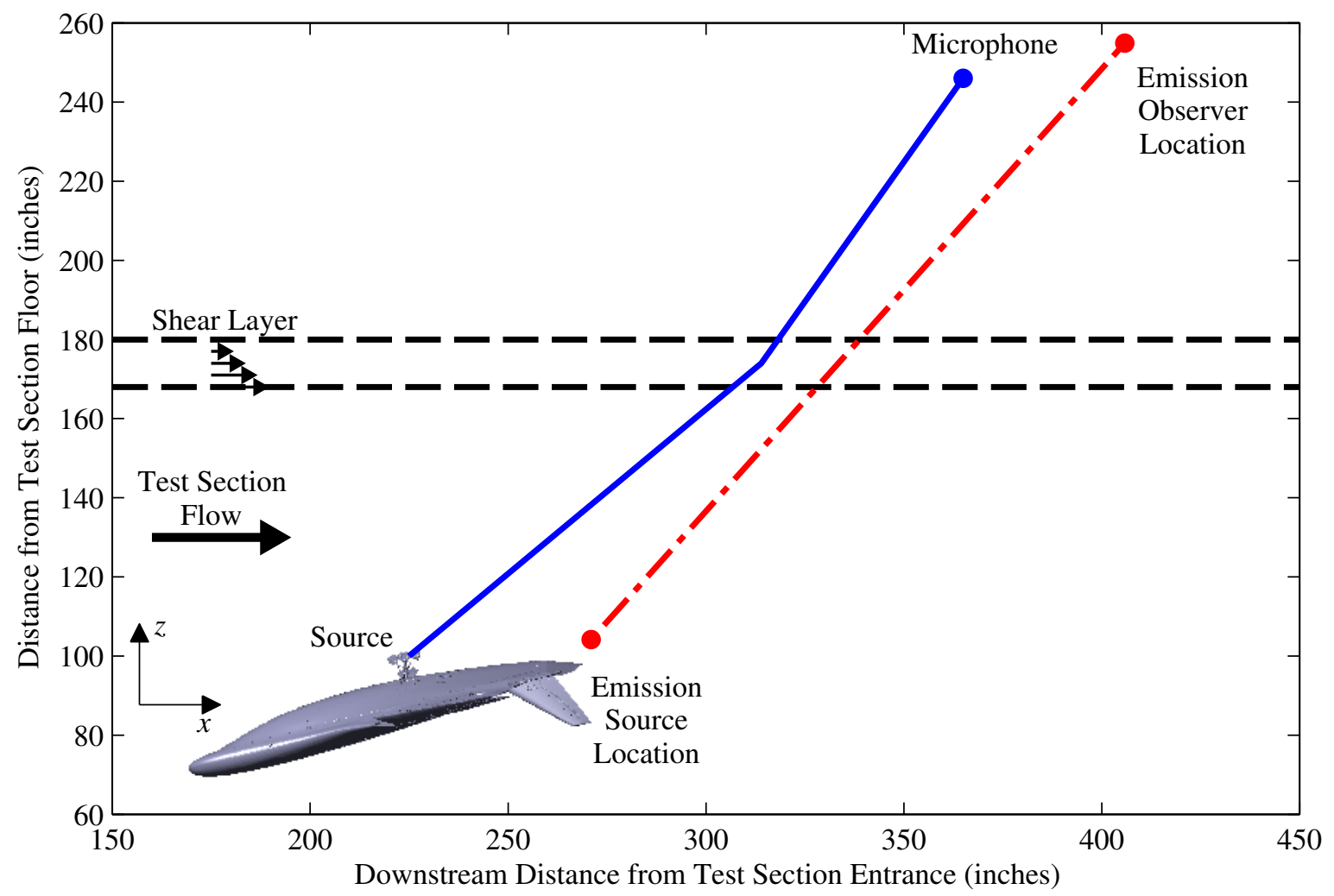

Figure 8. Example of shear layer correction applied to a microphone stationed 365 inches downstream from the test section entrance. The HWB model is at $14.5^{\circ}$ AoA, and the test section Mach number is $M=0.17$. 
free shear layers bounding the test section. The analysis procedure must determine which shear layer refracts the acoustic wave passing from the source to each microphone.

For the general 3-dimensional measurement geometry case, the set of equations in Amiet's21 Eq. A23 must be solved to locate the shear layer intersection point of a given ray. However, a 2-dimensional approximation can be used. Here, the 3-dimensional coordinate system is rotated about the flow axis such that the geometric line connecting the source and the observer lies within the new $x-z$ plane. This rotation allows for the use of Eq. 1 from Amiet ${ }^{21}$ under the assumption that the $y$ intersection coordinate in the original coordinate system is co-planar with this rotated $x-z$ plane. Alternatively phrased, the ray does not bend in any direction but the flow direction. The benefit is that the solution of a single equation nonlinear root-finding problem is significantly less expensive than the solution of a root-finding problem involving a system of nonlinear equations. Additionally, this assumption automatically determines which shear layer is appropriate for each source-observer pairing by finding which shear layer intersects the new $x-z$ plane. Testing of this assumption for various measurement configurations shows it to have minimal impact on the emission coordinates, ray direction and corrected levels with respect to the overall scale of the problem.

This procedure outputs an acoustic level correction, as well as a model of the geometry of the acoustic ray path and emission coordinates. Amiet's method corrects the data such that the emission path length is equal to the direct source-observer path length (as opposed to the refracted path length). Knowledge of the ray direction and emission path length is necessary for subsequent corrections, thus the shear layer correction is computed prior to the corrections for atmospheric attenuation and directivity. Emission coordinates are logged for use in the analyses reported in companion papers. ${ }^{5}+7$

\section{IV.C. Atmospheric Attenuation}

For full-scale analysis of an HWB aircraft, model-scale data are desired up to $80 \mathrm{kHz}$. For source-observer distances on the scale of this test, atmospheric attenuation of the acoustic energy may be significant. A lossless acoustic "measurement" is often desired as an interim step for subsequent applications, such as model scaling and re-propagation calculations. A model of the attenuation loss may be applied to estimate this equivalent lossless propagated measurement. A standard for computing energy loss for a given frequency and distance as a function of atmospheric properties exists $\underset{22}{22}$ and is directly implemented and applied to the post-processed data. This standard provides the attenuation as a function of frequency, atmospheric pressure, temperature, and humidity. An example set of attenuation calculations is shown in Fig. 9 for a given atmospheric pressure and temperature. The plotted values have an uncertainty of $\pm 10 \%$ at higher frequencies according to the standard ${ }^{22}$ The curves show attenuation in units of $\mathrm{dB} /$ inch, so at higher frequencies corrections of upwards of $10 \mathrm{~dB}$ may be applied to the data. The correction uses the emission path length provided by the shear layer correction calculation.

\section{IV.D. Microphone Directivity}

Microphones are not ideal signal observers, and have an influence on the recorded acoustic field determined by their installation characteristics ${ }^{23}$ The test was conducted with protective grids removed from the microphones (hence no grid correction is required), but the scattering microphone installation geometry must still be considered in the correction process. For free field microphone installation of the Brüel \& Kjær 4138 1/8" pressure field microphones mounted along the sideline towers and overhead truss, a directivity correction table was provided by the manufacturer as a function of angle of incidence from the normal of the microphone face, $\theta$, and frequency. This correction was applied with interpolation to the appropriate angles and frequencies. For most acquisitions, this correction was significantly greater than the corresponding pressure field correction for a given angle and frequency.

For pressure field microphone installation, such as the Brüel \& Kjær 4938 1/4" pressure field microphones installed in the array plate, the majority of the installation influence is dealt with at the CSM construction phase when the signal level is divided by 2, approximating the installation as an infinite baffle. However, at higher frequencies, some wave averaging occurs over the face of the microphone diaphragm, and must be modeled. One simple way to model the microphone is as a flexible membrane installed in an infinite baffle. An analytic solution exists for the far field directivity of a radiating, simply supported circular membrane in an infinite baffle, 24 and is given by

$$
D(\theta)=\frac{8 J_{2}(k R \sin \theta)}{(k R \sin \theta)^{2}} .
$$




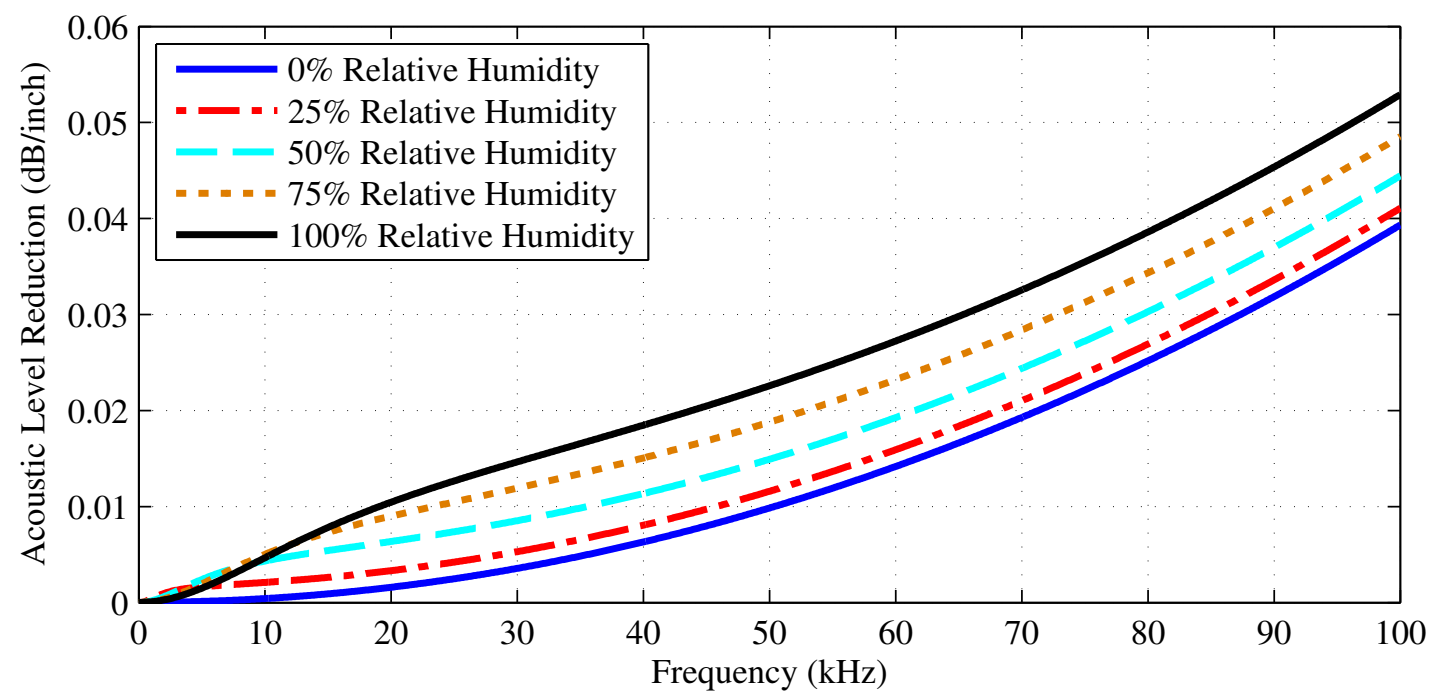

Figure 9. Example of atmospheric attenuation as a function of frequency and humidity, for atmospheric pressure of $101.3 \mathrm{kPa}$ and temperature of $273 \mathrm{~K}$.

Here, $k$ is the acoustic wavenumber computed using the speed of sound in humid air ${ }^{25} R$ is the membrane radius, and $J_{2}$ is the second order Bessel function of the first kind. Via reciprocity, the directivity pattern of a radiating, simply supported circular membrane should equate to the receptivity of a microphone diaphragm due to a source located at a given angle. Thus, the directivity function of Eq. (14) can be used to correct flush-mounted microphone levels by dividing the pressure value by $D$. It should be noted that as this formulation has a singularity at $\theta=0^{\circ}$, a refactoring may be preferable. Through a series of Bessel function identities, 26 Eq. (14) can be re-stated as

$$
D(\theta)=J_{0}(k R \sin \theta)+\frac{4}{3} J_{2}(k R \sin \theta)+\frac{1}{3} J_{4}(k R \sin \theta),
$$

which, assuming access to good Bessel function libraries, avoids the computational trap for small $\theta$. $\theta$ is determined by the ray path computed in the shear layer correction calculation, in conjunction with the microphone orientation. As mentioned previously, for most acquisitions this correction was significantly smaller than the corresponding free field correction for a given angle and frequency.

An example of the importance of these directivity corrections is shown in Fig. 10. This figure shows a comparison of autospectral measurements for the Broadband Engine Noise Simulators (BENS) ${ }^{5}$ Here, the array plate was located such that the array center microphone was approximately normal to the axis of BENS exhaust, with no tunnel flow, and data were acquired for BENS operation. The acoustic hardware was then traversed such that the truss center microphone was located where the array center microphone had been located, and the measurement repeated. For the uncorrected data, recalling that the pressure doubling on the array face has already been removed, there is a significant difference between the two spectral estimates. However, when directivity effects are taken into account for both microphones, the spectra show agreement due to the significant shift of the truss microphone data from the free field correction. As expected based on the formulation, for a near-zero observation angle little correction occurs to the array microphone data.

\section{Summary}

An outline of the data processing methodology for the HWB acoustic test in NASA Langley's 14- by 22-Foot Subsonic Wind Tunnel is provided. The procedure for taking the data from the raw integer count stream recorded by the DAS to acoustic CSMs is shown as a step-by-step process, and discussed in terms of practical implementation. Existing code was updated from a legacy cluster Fortran code to a MATLAB program utilizing GPU computing, providing over an order of magnitude reduction in processing time and allowing for in-situ data analysis. Post-test modifications to the CSM construction process are reported. 


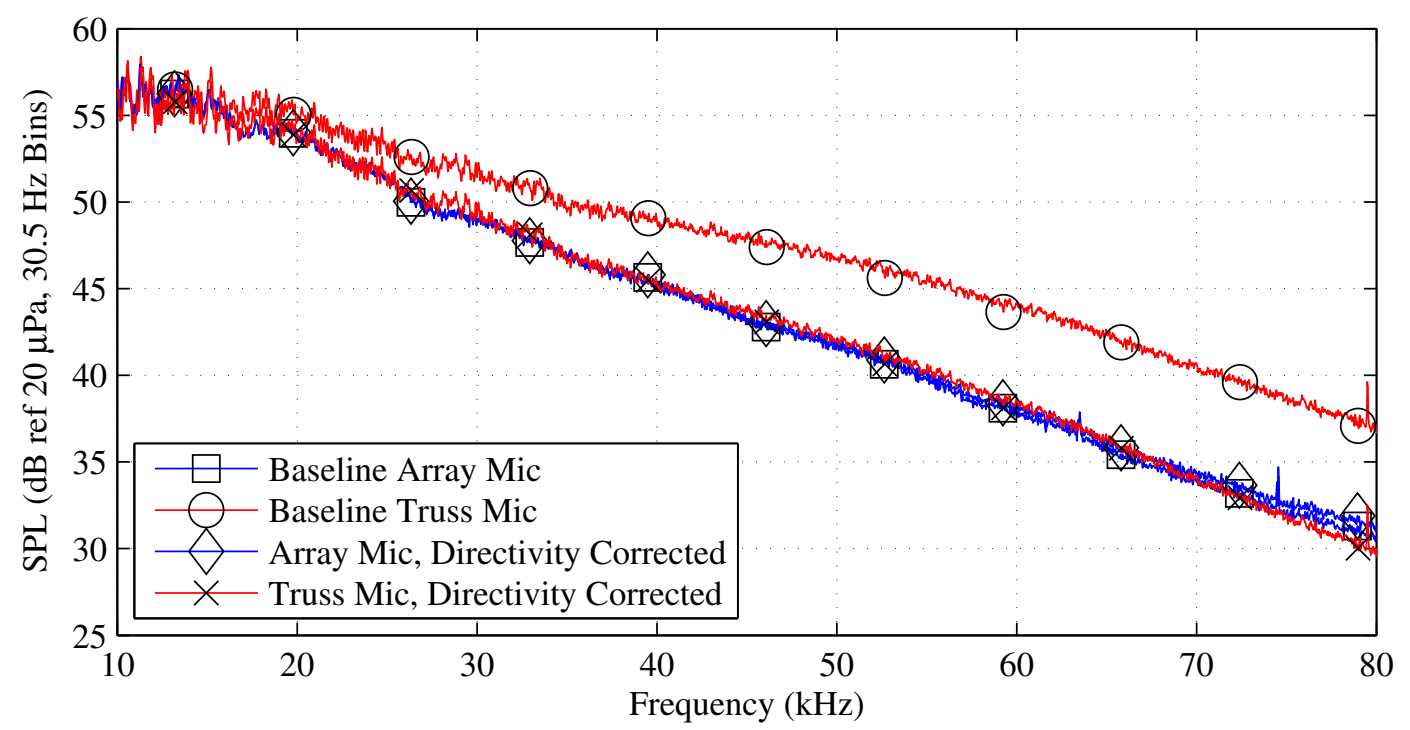

Figure 10. Comparison of spectra for array and truss microphones approximately normal to the axis of BENS exhaust, without and with directivity correction. For this comparison, the free field correction applied to the truss microphone spectrum is the dominant factor in the data collapse.

A novel transient rejection method is presented. The method analyzes a time series for transient bursts, and removes data blocks contaminated by the bursts. The method operates under the assumption that the desired signal to retain is a band-limited, stationary random process with lower block variance than the transient bursts to be removed. The method demonstrates the ability to separate contaminated blocks from uncontaminated ones. It is implemented as a completely automated process, with safeguards imposed should an aberrant signal show statistical behavior which causes the method to reject too much data. The method outputs CSMs, passed on to other analysis techniques, or autospectra, used with a baseline set of data corrections.

Individual corrections to autospectral data are discussed, along with the assumptions involved in their use and the inherent limitations. Background subtraction can help remove facility background acoustics as well as electronic noise in the microphone measurements, under the assumption that the background noise is statistically independent from the acoustic sources of interest and stationary from acquisition to acquisition. Shear layer correction accounts for acoustic wave refraction through a shear layer by modeling the layer as a plane and solving an appropriate set of equations. A 2-D collapse of the problem reduces the set of equations to a single equation, accelerating analysis. Atmospheric attenuation reduces observed acoustic levels and is considered significant for the scales of this test. It is corrected by using an existing ANSI standard, under the assumption that the mean test section flow has little influence on the attenuation characteristics. Microphone measurement directivity corrections are performed using either supplied manufacturer data or an analytic model. A simple test case demonstrates expected collapse of the data between microphone installations when microphone directivity corrections are applied.

The final product of these correction procedures is a set of lossless auto spectra corrected for observer interference, provided in emission coordinates at model scale. The data can then be used to perform a noise component analysis or the overall system-level noise assessment of the HWB N2A-EXTE.

\section{Acknowledgments}

The authors would like to acknowledge the support provided by the 14- by 22- Foot Subsonic Tunnel team and by colleagues in the Aeroacoustics and Advanced Sensing \& Optical Measurements branches at NASA Langley Research Center. This work was funded by the NASA Environmentally Responsible Aviation Project. 


\section{References}

${ }^{1}$ Collier, F. S., "Environmentally Responsible Aviation (ERA) Project," presentation, NASA Fundamental Aeronautics Program, Third Annual Meeting, Atlanta, Georgia, September 29 - October 12009.

${ }^{2}$ Heath, S. L., Brooks, T. F., Hutcheson, F. V., Doty, M. J., Haskin, H. H., Spalt, T. B., Bahr, C. J., Burley, C. L., Bartram, S. M., Humphreys, W. M., Lunsford, C. B., Popernack, T., Colbert, S., Hoad, D., Becker, L. E., Stead, D. J., Kuchta, D., and Yeh, L., "Hybrid Wing Body Aircraft Acoustic Test Preparations and Facility Upgrades," AIAA-2013-2623, 28th AIAA Aerodynamic Measurement Technology, Ground Testing, and Flight Testing Conference, San Diego, California, June 2013.

${ }^{3}$ Spalt, T. B., Brooks, T. F., Bahr, C. J., Plassman, G. E., Becker, L. E., and Stead, D. J., "Calibrations of the NASA Langley 14- by 22-Foot Subsonic Tunnel in Acoustic Configuration," accepted, 20th AIAA/CEAS Aeroacoustics Conference, Atlanta, Georgia, June 2014.

${ }^{4}$ Humphreys, W. M., Brooks, T. F., Bahr, C. J., Spalt, T. B., Bartram, S. M., Culliton, W. G., and Becker, L. E., "Development of a Microphone Phased Array Capability for the Langley 14- by 22-Foot Subsonic Tunnel," accepted, 20th AIAA/CEAS Aeroacoustics Conference, Atlanta, Georgia, June 2014.

${ }^{5}$ Hutcheson, F. V., Brooks, T. F., Burley, C. L., Bahr, C. J., Stead, D. J., and Pope, D. S., "Shielding of Turbomachinery Noise from a Hybrid Wing Body Aircraft Configuration," accepted, 20th AIAA/CEAS Aeroacoustics Conference, Atlanta, Georgia, June 2014 .

${ }^{6}$ Doty, M. J., Brooks, T. F., Burley, C. L., Bahr, C. J., and Pope, D. S., "Jet Noise Shielding Provided by a Hybrid Wing Body Aircraft," accepted, 20th AIAA/CEAS Aeroacoustics Conference, Atlanta, Georgia, June 2014.

${ }^{7}$ Burley, C. L., Brooks, T. F., Hutcheson, F. V., Doty, M. J., Lopes, L. V., Nickol, C. L., Vicroy, D. D., and Pope, D. S., "Noise Scaling and Community Noise Metrics for the Hybrid Wing Body Aircraft," accepted, 20th AIAA/CEAS Aeroacoustics Conference, Atlanta, Georgia, June 2014.

${ }^{8}$ Heath, S. L., Brooks, T. F., Hutcheson, F. V., Doty, M. J., Bahr, C. J., Hoad, D., Becker, L. E., Humphreys, W. M., Burley, C. L., Stead, D. J., Pope, D. S., Spalt, T. B., Kuchta, D., Plassman, G. E., and Moen, J., "NASA Hybrid Wing Body Aircraft Aeroacoustic Test Data Report," NASA TM (TBD), NASA - Environmentally Responsible Aviation Project, 2014.

${ }^{9}$ Rew, R., Davis, G., Emmerson, S., Davies, H., Hartnett, E., Heimbigner, D., and Fisher, W., "The NetCDF Users Guide," September 2013

${ }^{10}$ National Instruments Corporation, LabVIEW 8.5, Austin, Texas.

${ }^{11}$ The MathWorks, Inc., MATLAB and Parallel Computing Toolbox Release 2012b, Natick, Massachusetts.

${ }^{12}$ Bendat, J. S. and Piersol, A. G., Random Data Analysis and Measurement Procedures, chap. 5, John Wiley \& Sons, Inc., New York, NY, 3rd ed., 2000, p. 140.

${ }^{13}$ Zelen, M. and Severo, N. C., "Probability Functions," Handbook of Mathematical Functions, edited by M. Abramowitz and I. A. Stegun, chap. 26, Dover Publications, Inc., New York, 1972, pp. 940-943.

${ }^{14}$ Bendat, J. S. and Piersol, A. G., Random Data Analysis and Measurement Procedures, chap. 8, John Wiley \& Sons, Inc., New York, NY, 3rd ed., 2000, p. 311.

151.3.6.6.11. Gamma Distribution, NIST/SEMATECH e-Handbook of Statistical Methods, http://www.itl.nist.gov/div898/handbook/, October 2013.

${ }^{16}$ Zhang, J., "Reducing bias of the maximum likelihood estimator of shape parameter for the gamma distribution," Computational Statistics, Vol. 28, No. 4, August 2013, pp. 1715-1724.

${ }^{17}$ Cardoso, J., "Infomax and Maximum Likelihood for Blind Source Separation," IEEE Signal Processing Letters, Vol. 4, No. 4, April 1997, pp. 112-114.

${ }^{18}$ Humphreys, W. M., Brooks, T. F., Jr., W. W. H., and Meadows, K. R., "Design and Use of Microphone Directional Arrays for Aeroacoustic Measurements," AIAA-98-0471, 36th AIAA Aerospace Sciences Meeting \& Exhibit, Reno, NV, January 1998.

${ }^{19}$ Hutcheson, F. V. and Brooks, T. F., "Measurement of Trailing Edge Noise Using Directional Array and Coherent Output Power Methods," AIAA-2002-2472, 8th AIAA/CEAS Aeroacoustics Conference, Breckenridge, CO, June 2002.

${ }^{20}$ Brooks, T. F. and Humphreys, W. M., "A deconvolution approach for the mapping of acoustic sources (DAMAS) determined from phased microphone arrays," Journal of Sound and Vibration, Vol. 294, 2006, pp. 856-879.

${ }^{21}$ Amiet, R. K., "Refraction of Sound by a Shear Layer," Journal of Sound and Vibration, Vol. 58, No. 4, 1978, pp. 467-482.

${ }^{22}$ Method for Calculation of the Absorption of Sound by the Atmosphere, ANSI S1.26-1995 (ASA 113-1995), Acoustical Society of America, 1995.

${ }^{23}$ Microphone Handbook, Vol. 1, Brüel \& Kjær, 1996.

${ }^{24}$ Blackstock, D. T., Fundamentals of Physical Acoustics, chap. 13, John Wiley \& Sons, Inc., New York, NY, 2000, p. 464.

${ }^{25}$ Cramer, O., "The variation of the specific heat ratio and the speed of sound in air with temperature, pressure, humidity, and $\mathrm{CO}_{2}$ concentration," Journal of the Acoustical Society of America, Vol. 93, No. 5, May 1993, pp. 2510-2516.

${ }^{26}$ Olver, F. W. J., "Bessel Functions of Integer Order," Handbook of Mathematical Functions, edited by M. Abramowitz and I. A. Stegun, chap. 9, Dover Publications, Inc., New York, 1972, p. 361. 